\title{
Uso de álcool entre adolescentes escolares: um estudo-piloto ${ }^{1}$
}

\author{
Luana Dallo ${ }^{2}$ \\ Universidade Estadual Paulista, Marília-SP, Brasil \\ Raul Aragão Martins \\ Universidade Estadual Paulista, São José do Rio Preto-SP, Brasil
}

\begin{abstract}
Resumo: O uso de álcool entre adolescentes é um tema que preocupa profissionais da saúde pelos prejuízos acarretados e pela displicência da sociedade quanto ao uso desta droga por essa faixa etária. Este artigo analisa o consumo de álcool entre alunos do ensino médio de três escolas de Cascavel-PR. O instrumento utilizado foi o Alcohol Use Disordens Identification Test (AUDIT), além de levantamento de informações sobre o nível socioeconômico, religião e o beber problemático de familiar. O resultado mostrou que $34,7 \%$ dos estudantes bebem de maneira arriscada, não havendo diferenças significativas por escola, gênero e nível socioeconômico. Ter familiar que bebe foi considerado fator de risco e ter religião, fator protetor. Esta pesquisa pode alertar a sociedade paranaense para a necessidade de estudos mais amplos, com o objetivo de desenvolver políticas públicas que incluam projetos de prevenção e intervenção dirigidos à população jovem.
\end{abstract}

Palavras-chave: adolescência, abuso de álcool, estudantes.

\section{Use of alcohol among adolescent students: a pilot test}

\begin{abstract}
The consumption of alcohol among adolescents is an issue that concerns health professionals given the harm it causes and the indifference of the society regarding its use in this age range. This study analyzed the use of alcohol among high school students in three schools in Cascavel, PR, Brazil. The Alcohol Use Disorders Identification Test (AUDIT) was used and data concerning socioeconomic information, religion, and whether there was abusive consumption of alcohol in the family were also collected. The results revealed that $34.7 \%$ of the students present abusive consumption. No statistically significant differences were found concerning schools, gender, and socioeconomic conditions. Having a family member who consumes alcohol was considered a risk factor and having a religion was considered a protection factor. This study shows the need of further studies to warn the society in Paraná aiming the development of public policies including prevention and intervention projects directed to adolescents.
\end{abstract}

Keywords: adolescence, alcohol abuse, students.

\section{Uso del alcohol entre adolescentes escolares: un estudio-piloto}

\begin{abstract}
Resumen: El uso de alcohol por adolescentes es un tema que preocupa profesionales de la salud por los perjuicios acarreados y la asignatura de la sociedad en cuanto al uso de esta droga por ese rango de edad. Este estudio investigó el consumo de alcohol entre alumnos de secundaria de tres escuelas de Cascavel-PR, Brasil. El instrumento utilizado fue el Alcohol Use Disorders Identification Test (AUDIT), además de nivel socioeconómico, religión y beber problemático de familiar. El resultado mostró que el 34,7\% de los estudiantes beben peligrosamente, no habiendo diferencias significativas por escuela, género y nivel socioeconómico. Tener familiar que bebe fue considerado factor de riesgo y tener religión factor protector. Esta investigación puede alertar la sociedad paranaense para la necesidad de estudios más amplios, con el objetivo de desarrollar políticas públicas que incluyan proyectos de prevención e intervención dirigidos a la población joven.
\end{abstract}

Palabras clave: adolescencia, abuso de alcohol, estudiantes.

Uso de álcool por adolescentes é um tema investigado por pesquisadores preocupados com o seu início nessa faixa etária e com estratégias de prevenção. Estudos indicam (Dallo, 2009; Galduróz, Noto, Fonseca, \& Carlini, 2005; Martins, Cruz, Teixeira, \& Manzato, 2008) que o álcool é a primeira droga a ser usada, e, geralmente, o uso excessivo dessa droga é a porta de entrada ao consumo das outras. Dados brasileiros apontam relação direta do álcool com atividade

${ }^{1}$ Este texto foi revisado seguindo o Acordo Ortográfico da Língua Portuguesa (1990), em vigor a partir de $1^{\circ}$ de janeiro de 2009.

${ }^{2}$ Endereço para correspondência:

Luana Dallo. Rua Professor Assis Gonçalves, 1506. CEP 80.020-250.

Curitiba-PR, Brasil. E-mail: luana@aol.com.br sexual precoce e sem uso de preservativo, violência, acidentes de trânsito e quedas no desempenho escolar (Laranjeira, Pinsky, Zaleski, \& Caetano, 2007; Martins, 2006).

Uma importante sequência de estudos epidemiológicos (Carlini, Carlini-Cotrim, Silva Filho \& Barbosa, 1989, 1990; Galduróz e cols., 2005; Galduróz, Noto, \& Carlini, 1997), feitos em diversas cidades brasileiras com estudantes do ensino fundamental e médio, apontou o álcool como a droga mais consumida e com início precoce. No I Levantamento do Padrão de Uso de Álcool (Laranjeira e cols., 2007), as informações foram separadas por faixa etária com o objetivo de visualizar como bebem os adultos (maiores de 18 anos) e os adolescentes (de 14 a 17 anos). Isso possibilitou observar que os adultos jovens (de 18 a 25 anos) apresentaram uma média de idade de início de uso de 15,3 anos e de consumo 
regular de 17,3 anos, enquanto os adolescentes (de 14 a 17 anos) iniciaram o uso em média com 13,9 anos e o consumo regular com 14,6 anos. Essas diferenças foram significativas, indicando que o início do uso e o consumo regular de álcool vêm ocorrendo mais precocemente.

Um estudo (Fiorini, Alvesa, \& Ferreira, 2003), realizado com 1.500 estudantes de uma universidade pública e outra particular da cidade de Alfenas-MG, mostrou que 55\% dos estudantes usavam algum tipo de droga. Entretanto, os estudantes revelaram que já consumiam álcool e tabaco antes de ingressar na universidade, indicando que o início das drogas lícitas ocorre quando estão no ensino médio e continua com o ingresso na universidade.

Segundo Scivolleto e Giusti (2007), a curiosidade natural do adolescente influencia na experimentação de substâncias psicoativas, o que o impulsiona a experimentar novas sensações e prazeres. O jovem vive o presente e busca realizações imediatas, e os efeitos das drogas vão ao encontro desse perfil, proporcionando prazer passivo e imediato.

O agravo em relação ao aumento do uso ocorre por incentivo social, quando a embriaguez representa prova de força ou resistência entre os amigos, por funcionar como agente socializador entre familiares e pares, presentes em eventos sociais como festas de 15 anos; por meio da divulgação das bebidas alcoólicas pela mídia, focada no público jovem; e o não cumprimento e fiscalização precária da Lei no. 8.069 (1990) que proíbe a venda aos menores de 18 anos.

Pesquisas sobre o contexto de uso de álcool por adolescentes (Cruz, 2006; Dallo, 2009; Martins, 2006) apontam que o início do uso ocorre, na maioria das vezes, entre familiares e depois em festas e com amigos. A bebida preferida é a cerveja, e, dificilmente, os adolescentes bebem sozinhos. As propagandas nas mídias também mostram jovens bebendo em festas, bares, praias e, geralmente, em grupos de amigos.

O documento Política do Ministério da Saúde para a atenção integral a usuários de álcool e outras drogas (Ministério da Saúde, 2004) enfatizou o álcool como o maior problema de saúde pública, apontando que, no ano de 2001, o custo anual em internações para o SUS foi mais de $60 \mathrm{mi}$ lhões de reais, não incluindo os gastos com tratamento ambulatorial, nem com outros modos de internações e tratamento das doenças indiretamente provocadas pelo consumo do álcool. Segundo essa política, a reversão do quadro é possível via realização de pesquisas científicas, prevenção precoce e intervenções breves. O mesmo documento destacou, quanto ao planejamento de ações preventivas relativas ao uso de álcool e outras drogas, a importância de minimizar a influência de fatores de risco para o uso do álcool e, da mesma forma, o reforço para os fatores protetores.

Os fatores de risco para o uso de álcool e outras drogas são características ou atributos de um indivíduo, grupo ou ambiente de convívio social que contribuem para o aumento da probabilidade da ocorrência do uso, enquanto os fatores protetores contribuem para a diminuição da perspectiva de uso. Os fatores de risco e de proteção podem ser identificados em todos os domínios da vida: nos próprios indivíduos, em suas famílias, pares, escolas e comunidade.

Fundamentado na política pública do Ministério da Saúde e nos principais estudos brasileiros sobre o uso de álcool e outras drogas, o objetivo deste estudo foi conhecer o padrão do uso de álcool por alunos de três escolas (duas públicas e uma privada) do município de Cascavel-PR, como um projeto-piloto para a realização de futuras pesquisas no estado, com o propósito de efetuar medidas de prevenção baseadas na realidade paranaense.

\section{Método}

Realizou-se levantamento epidemiológico do consumo de álcool com estudantes do ensino médio de uma escola privada, uma pública localizada no centro e outra situada na periferia de Cascavel-PR, município do oeste deste estado, com cerca de 285 mil habitantes.

\section{Participantes}

Fizeram parte do estudo 133 estudantes matriculados no ano de 2007, no ensino médio de três escolas, sendo duas públicas e uma privada. As duas escolas públicas foram indicadas pelo escritório local da Secretaria de Educação como sendo representativas de instituições situadas no centro e na periferia de Cascavel. A escola privada foi escolhida por ser a que respondeu prontamente ao convite feito pelos pesquisadores entre as que funcionam na cidade. Foram convidados para participar do estudo os alunos do $1^{\circ}$ ano por formarem as salas com o maior número de estudantes. $\mathrm{Na}$ escola privada foram, também, convidados alunos do $2^{\circ}$ ano para comporem um número proporcional ao das escolas públicas, no período diurno. No total foram 69 (51,9\%) alunos da escola pública localizada no centro, sendo $33(52,1 \%)$ do período diurno e 36 (48\%) do noturno. Na escola pública de periferia, foram $30(22,6 \%)$ e na privada $34(25,6 \%)$ alunos.

\section{Instrumentos}

Utilizou-se questionário autoaplicado e com identificação do participante, composto de dados sociodemográficos, histórico familiar e o Alcohol Use Disorders Identification Test (AUDIT) (Babor, Fuente, Sauders, \& Grant, 1992). Este instrumento foi desenvolvido por um grupo de pesquisadores, sob os auspícios da Organização Mundial da Saúde (OMS) e adaptado para o Brasil por Mendez (1999). Ele é composto por 10 questões, as três primeiras avaliam quantidade, frequência e embriaguez; as três seguintes, sintomas de dependência; e as quatro últimas, o risco de consequências danosas ao usuário. As respostas para as questões variam de "0" a "4", fazendo com que a pontuação máxima seja 40. Os estudantes são classificados como positivos, quando fazem 8 ou mais pontos no teste, o que corresponde a uso de risco. 
São classificados como negativos os que fazem uso moderado ou são abstêmios. A escolha deste teste para compor o levantamento inicial deu-se por ser de preenchimento rápido e já ter sido aplicado à população estudada (Cruz, 2006; Martins, Cruz e cols., 2008).

\section{Procedimento}

\section{Coleta de dados}

Inicialmente pediu-se permissão à direção das escolas para fazer a pesquisa, posteriormente foi entregue o Termo de Consentimento Livre e Esclarecido (TCLE), para que os alunos assinassem ou, no caso dos menores de 18 anos, pedissem autorização aos pais ou responsáveis. Finalmente, depois de recebido o TCLE com as autorizações, foi aplicado o questionário coletivamente nas salas de aula.

\section{Análise dos dados}

Os dados foram digitados em uma planilha eletrônica e, posteriormente, exportados a um programa de análises estatísticas denominado SPSS (Statistical Package for the social Science, 2003). Nesse programa foram computadas as frequências de cada questão, para depois realizar as análises, a partir dos cruzamentos entre as variáveis. Estabeleceu-se o nível de 0,05 de significância para as comparações realizadas com o teste de Qui-Quadrado (Pereira, 1999).

\section{Considerações éticas}

Antes de iniciar a coleta de dados, a pesquisa foi submetida ao Comitê de Ética em Pesquisa da Faculdade de Filosofia e Ciências da Universidade Estadual Paulista (UNESP) e aprovada em 28 de novembro de 2007 (Parecer $\left.n^{\circ} 2325 / 2007\right)$. Os participantes foram informados, antecipadamente, de que poderiam desistir de participar da pesquisa a qualquer momento, pois a participação é de livre consentimento. Além disso, foi informado aos alunos e à direção/ coordenação das escolas que dados individuais não seriam fornecidos, por se tratar de material sigiloso. Considerou-se, assim, não haver riscos de prejuízos psicológicos aos participantes da pesquisa, pois o objetivo é apenas avaliar o uso de álcool. Informou-se, porém, que, se acontecesse alguma manifestação de sofrimento por parte dos sujeitos pesquisados, a aplicação da pesquisa seria suspensa, e o método reelaborado.

\section{Resultados e Discussão}

Os resultados desta pesquisa referem-se a um estudopiloto por haver pouca abrangência populacional, não sendo possível considerar os dados como representativos da população de Cascavel. Apesar dessa limitação, estas informações não devem ser negligenciadas, pois apontaram números significativos, destacando-se a importância de realizar mais pesquisas nessa região.

O levantamento possibilitou o cruzamento de informações sobre o perfil sociodemográfico dos participantes de cada escola e o padrão de bebidas alcoólicas. Em relação às características sociodemográficas, a maioria dos estudantes era adolescente, representando $92,5 \%$ com idade média de 14 a 18 anos. A participação foi semelhante em ambos os gêneros, $49,6 \%$ eram do sexo feminino e $50,4 \%$, do masculino. Do total de alunos, 72,2\% tinham nível socioeconômico B e C, de acordo com o critério ABA/ABIPEME (Almeida \& Wickerhauser, 1991), 54,8\% se denominaram católicos e $19,5 \%$, evangélicos. Na escola pública localizada no centro foi avaliada a variável "período", $72,9 \%$ dos alunos estudavam no período da manhã e 27,1\% estudavam à noite (Tabela 1).

Tabela 1

Frequência e porcentagem do resultado da escala AUDIT por escola, gênero, periodo e nível socioeconômico

\begin{tabular}{|c|c|c|c|c|c|c|}
\hline & \multicolumn{2}{|c|}{ Positivos } & \multicolumn{2}{|c|}{ Negativos } & \multicolumn{2}{|c|}{ Total } \\
\hline & $f$ & $\%$ & $f$ & $\%$ & $f$ & $\%$ \\
\hline \multicolumn{7}{|l|}{ Escola } \\
\hline Pública periferia & 11 & 36,7 & 19 & 63,3 & 30 & 22,5 \\
\hline Pública centro & 20 & 29 & 49 & 71 & 69 & 51,8 \\
\hline Privada & 15 & 44,1 & 19 & 55,9 & 34 & 25,5 \\
\hline \multicolumn{7}{|l|}{ Sexo } \\
\hline Feminino & 21 & 31,8 & 45 & 68,2 & 66 & 49,6 \\
\hline Masculino & 25 & 37,3 & 42 & 62,7 & 67 & 50,4 \\
\hline \multicolumn{7}{|l|}{ Período } \\
\hline Diurno & 33 & 34 & 64 & 66 & 97 & 72,9 \\
\hline Noturno & 13 & 36,1 & 23 & 63,9 & 36 & 27,1 \\
\hline
\end{tabular}


Tabela 1 - (continuação)

\begin{tabular}{|c|c|c|c|c|c|c|}
\hline & \multicolumn{2}{|c|}{ Positivos } & \multicolumn{2}{|c|}{ Negativos } & \multicolumn{2}{|c|}{ Total } \\
\hline & $f$ & $\%$ & $f$ & $\%$ & $f$ & $\%$ \\
\hline \multicolumn{7}{|l|}{ Nível socioeconômico } \\
\hline A & 8 & 40 & 12 & 60 & 20 & 15,7 \\
\hline $\mathrm{B}$ & 20 & 31,3 & 44 & 68,8 & 64 & 50,4 \\
\hline $\mathrm{C}$ & 13 & 40,6 & 19 & 59,4 & 32 & 25,2 \\
\hline $\mathrm{D}$ & 3 & 27,3 & 8 & 72,7 & 11 & 8,7 \\
\hline \multicolumn{7}{|l|}{ Religião } \\
\hline Católico & 28 & 38,4 & 45 & 61,6 & 73 & 100 \\
\hline Evangélico & 2 & 7,7 & 24 & 92,3 & 26 & 100 \\
\hline Em branco/não tem & 12 & 44,4 & 15 & 55,6 & 27 & 100 \\
\hline Outras & 4 & 57,1 & 3 & 42,9 & 7 & 100 \\
\hline
\end{tabular}

O número de estudantes identificados como bebedores de risco, nas três escolas nesta amostra pequena, foi de $34,6 \%$, número relativamente alto, se comparado a dados de uma pesquisa realizada com estudantes do ensino médio de uma cidade do interior paulista, na qual foi constatado o índice de 22,3\% (Martins, Manzato, Cruz, Poiate, \& Scarin, 2008).

Esse índice médio das três escolas, ao ser desdobrado em cada uma, apresentou que $36,7 \%$ do total dos alunos são positivos na escola pública localizada na periferia da cidade; $29 \%$ na escola pública no centro da cidade e $44,1 \%$ na escola privada. Apesar de a escola privada ter apresentado um número maior de adolescentes considerados positivos, não houve diferença significativa entre as três instituições, conforme o teste do Qui-Quadrado $\left(\chi^{2}=2,359, p=0,304\right)$.

Quanta à variável gênero, $31,8 \%$ das moças e 37,3\% dos rapazes apresentaram um beber arriscado. Embora alguns estudos sobre o padrão do beber apresentassem o sexo masculino como fator de risco (Cruz, 2006; Galduróz, Noto, \& Nappo, 2002), os dados desta pesquisa demonstraram que a frequência de uso de risco entre as moças foi próxima à dos rapazes. Isso se torna preocupante porque as mulheres apresentam alguns fatores que as tornam mais vulneráveis aos efeitos do álcool, como menos desidrogenase (metabolizador do álcool), menor peso, mais gordura corporal que retém o álcool, por mais tempo, e as variações do ciclo hormonal (Martins, 2006).

Estudiosos (Laranjeira \& Pinsky, 2000) alertam que essa mudança no comportamento do beber das mulheres pode ser devido à mudança no status e na sua participação na sociedade, pois em décadas passadas não era socialmente aceitável que a mulher exagerasse ou perdesse o controle sobre os seus atos.

Quanto ao período, diurno ou noturno, foi avaliado apenas na escola pública central, e o resultado foi que $34 \%$ dos alunos no turno da manhã e $36,1 \%$ da noite apresentaram um beber arriscado, não havendo diferenças significativas entre os períodos.

A maioria dos estudantes que participaram da pesquisa cursava o $1^{\circ}$ ano, pois apenas na escola privada uma turma do $2^{\circ}$ ano foi também incluída. Mesmo assim, os cálculos foram realizados e encontraram-se $47,1 \%$ de alunos positivos no $2^{\circ}$ ano e $32,8 \%$ no $1^{\circ}$. Supõe-se que essa diferença indique um aumento de uso no decorrer dos anos, ou seja, o aluno que já bebia no $1^{\circ}$ ano possivelmente aumentará o uso, quando estiver no segundo.

Os dados do nível socioeconômico não apontaram diferenças significativas, segundo o $\chi^{2}=1,349, p=0,718$, muitos estudantes desta pesquisa, pertencentes do nível A ao D, fazem uso da bebida excessivamente. Esse dado também é encontrado por outros pesquisadores (Baus, Kupek, \& Pires, 2002; Muza, Bettiol, Muccilo, \& Barbieri, 1997).

Outra característica importante foi que estudantes de denominação religiosa evangélica destacam-se no consumo controlado do álcool, sendo apenas $7,7 \%$ positivos do total de alunos evangélicos, enquanto $38,4 \%$ dos católicos, $44,4 \%$ dos que disseram não ter religião ou não informaram ou, ainda, $57,1 \%$ dos estudantes que responderam praticar outra religião foram positivos. Apesar das dificuldades para estabelecer um padrão medidor da religiosidade, durante os últimos 30 anos, dados quantitativos vêm apontando a relevância da religião na prevenção do consumo de drogas (Sanches \& Nappo, 2007). Estudos feitos em diversos contextos socioculturais demonstraram que, em estudantes adolescentes e jovens, o fato de não ter religião (ou pertencer a denominações mais liberais), ter pouca crença religiosa, não frequentar igreja e cultos, associa-se com maior uso de álcool e outras drogas (Dalgalarrondo, Soldera, Correa Filho, \& Silva, 2004).

Possuir um familiar bebedor foi descrito por autores (Silva e cols., 2003) como um preditivo de risco aumentado para o uso do álcool. Segundo dados obtidos nesta análise, $38,2 \%$ dos positivos apresentaram familiar que bebe excessivamente e $61,8 \%$ dos negativos. A partir desses resultados, pode-se supor que o fator de risco não é só para o abuso do álcool, mas para outras drogas e sofrimento psíquico. Também se deve considerar a resiliência como fator protetor e a razão para os adolescentes que, mesmo tendo familiar bebedor, não desenvolveram uso abusivo de álcool (Sapienza \& Pedremonico, 2005). 


\section{Considerações finais}

Percebeu-se que muitos participantes desta pesquisa bebem excessivamente, não havendo diferenças significativas entre classes sociais, sexo e escola, o que significa uso demasiado entre esses adolescentes e pode ser devido ao fato de o álcool ser uma droga lícita, de fácil acesso, custo baixo, além de ser divulgado por propagandas atraentes.

O instrumento AUDIT, aplicado nesta pesquisa, tem se mostrado uma ferramenta importante no rastreamento do uso de bebidas alcoólicas nessa população, por avaliar com precisão e rapidez o grau de comprometimento dos jovens com o álcool, além de ser um teste fácil e com boa aceitação entre estudantes do ensino médio (Martins, Manzato e cols., 2008).

Levantamentos epidemiológicos como este podem auxiliar na construção de políticas públicas em relação ao álcool, principalmente com o objetivo da prevenção, numa ótica que envolve três aspectos: biológicos, psicológicos e ambientais. Entende-se que o uso excessivo e a dependência do álcool não são determinados apenas por aspectos biológicos, o que dificulta a possibilidade da prevenção, mas também por aspectos psicológicos e ambientais, passíveis de serem mudados.

Canoletti e Soares (2005) fizeram uma análise da produção científica de 1991 a 2001 sobre os programas de prevenção ao consumo de drogas no Brasil e detectaram que, nos bancos de dados, apesar de se levantar uma quantidade significativa de artigos, somente uma parcela bastante reduzida diz respeito ao desenvolvimento de projetos de prevenção. Além disso, os autores apontaram que menos da metade dos programas analisados fez referência a um estudo epidemiológico preliminar, estando os demais projetos descompassados entre a realidade local e os objetivos e estratégias.

O Ministério da Saúde (2004) salienta que uma das questões importantes em relação à prevenção é considerar os fatores de risco e de proteção ao uso de drogas. Conhecendo-se tais fatores, as estratégias podem ser voltadas para a redução de fatores de vulnerabilidade e risco específicos e para o fortalecimento dos fatores de proteção. Implicando, necessariamente, na inserção comunitária das práticas propostas, com a colaboração de todos os segmentos sociais disponíveis.

Finalizando, apresentam-se três comentários. O primeiro refere-se aos estudos epidemiológicos realizados no Brasil, indicando o uso excessivo de álcool por adolescentes. Os resultados desta pesquisa, realizada em Cascavel, interior do Paraná, embora apresente uma amostra pequena, principalmente por haver uma grande porcentagem de bebedores de alto risco, apontam a necessidade de mais estudo nesse estado. O segundo, a importância dos levantamentos epidemiológicos por regiões, com o objetivo de conhecer a realidade local para a implantação de projetos de prevenção, salientando-se a eficácia do instrumento AUDIT para esse fim. E, por último, há a necessidade do desenvolvimento de programas de prevenção condizentes com os levantamentos epidemiológicos brasileiros.

\section{Referências}

Almeida, P. M., \& Wickerhauser, H. (1991). O critério ABA/ ABIPEME: Em busca de uma atualização. São Paulo: LPM/Burke.

Babor, T. F., Fuente, J. R., Saunders, J., \& Grant, M. (1992). The alcohol use disordens identification test: Guidelines for use in primary health care [Versão eletrônica]. Genebra: World Health Organization, 92(4), 1-29. Recuperado em 05 agosto 2009, de http://bases.bireme. br/cgibin/wxislind.exe/iah/online/?IsisScript=iah/iah.xis $\& \mathrm{src}=$ google $\&$ base $=$ PAHO\&lang $=$ p\&nextAction $=$ lnk $\&$ exprSearch $=14305 \&$ index

Baus, J., Kupek, E., \& Pires, M. (2002). Prevalência e fatores de risco relacionados ao uso de drogas entre escolares. Revista de Saúde Pública, 36(1), 40-46.

Canoletti, B., \& Soares, C. B. (2005). Drug consumption prevention programs in Brazil: Analyzis of the scientific production from 1991 to 2001 [Versão eletrônica]. Interface: Comunição, Saúde, Educação, 9(16), 115-129. Recuperado em 13 julho 2009, de http://www.scielo.br/ scielo.php?pid $=$ S141432832005000100010\&scrit $=$ sc i_abstract\&tlng $=$ en

Carlini, E. A., Carlini-Cotrin, B., Silva-Filho, A. R., \& Barbosa, M. T. S. (1989). O uso de drogas psicotrópicas por estudantes de $1^{\circ}$ e $2^{\circ}$ graus da rede estadual, em dez capitais. São Paulo: Centro Brasileiro de Informações sobre Drogas.

Carlini, E. A., Carlini-Cotrin, B., Silva Filho, A. R., \& Barbosa, M. T. S. (1990). II levantamento nacional sobre o uso de psicotrópicos em estudantes de primeiro e segundo graus. São Paulo: Centro Brasileiro de Informações sobre Drogas.

Cruz, L. A. N. (2006). Uso de álcool e julgamento sóciomoral de estudantes do ensino médio. Dissertação de Mestrado não publicada, Universidade Estadual Paulista, Marília, SP.

Dalgarrondo, P., Soldera, M. A., Correa Filho, H. R., \& Silva, C. A. M. (2004). Religião e uso de drogas por adolescentes [Versão eletrônica]. Revista Brasileira de Psiquiatria, 26(2), 82-90. Recuperado em 19 junho 2009, de http://www.scielo.br/pdf/rbp/v26n2/a04v26n2.pdf

Dallo, L. (2009). Padrão de uso de álcool e outras drogas entre estudantes de Cascavel-PR. Dissertação de Mestrado não publicada, Universidade Estadual Paulista, Marília, SP.

Fiorini, J. E., Alvesa, L., \& Ferreira, L. R. (2003). Uso de drogas lícitas e ilícitas no meio universitário de Alfenas [Versão eletrônica]. Revista do Hospital das Clínicas, 58(4), 199-206. Recuperado em 25 agosto 2009, de http://www.unifenas.br/PESQUISA/revistas/download/ ArtigosRev2_99/pag263-267.pdf 
Galduróz, J. C. F., Noto, A. R., \& Carlini E. A. (1997). IV levantamento sobre o uso de drogas entre estudantes de $1^{\circ}$ e $2^{\circ}$ graus em 10 capitais brasileiras. São Paulo: Centro Brasileiro de Informações sobre Drogas.

Galduróz, J. C. F., Noto, A. R., Fonseca, A. M., \& Carlini, E. A. (2005). V Levantamento nacional sobre o consumo de drogas psicotrópicas entre estudantes do ensino fundamental e médio da rede pública de ensino nas 27 capitais brasileiras. São Paulo: Centro Brasileiro de Informações sobre Drogas.

Galduróz, J. C. F., Noto, A. R., \& Nappo, S. A. (2002). I levantamento domiciliar sobre uso de drogas psicotrópicas no Brasil: Estudo envolvendo as 107 maiores cidades do país. São Paulo: Centro Brasileiro de Informações sobre drogas.

Laranjeira, R., \& Pinsky, I. (2000). Alcoolismo (6a ed.). São Paulo: Contexto.

Laranjeira, R., Pinsky, I., Zaleski, M., \& Caetano, R. (2007). I Levantamento nacional sobre os padrões de consumo de álcool na população brasileira. Brasília: Secretaria Nacional Antidrogas.

Lei No. 8.069, de 13 de julho de 1990. (1990, 13 de julho). Dispõe sobre o Estatuto da Criança e do Adolescente e dá outras providências. Congresso Nacional. Recuperado em 05 dezembro 2009, de http://www.planalto.gov.br/ ccivil/LEIS/L8069.htm

Martins, R. A. (2006). Uso de álcool, intervenção breve e julgamento sócio-moral em adolescentes que bebem excessivamente. Tese de Livre Docência não publicada, Universidade Estadual Paulista, São José do Rio Preto, SP.

Martins, R. A., Cruz, L. N., Teixeira, P. S., \& Manzato, A. (2008). Padrão de consumo de álcool entre estudantes do ensino médio de uma cidade do interior do estado de São Paulo [Versão eletrônica]. SMAD: Revista Eletrônica Saúde Mental Álcool e Drogas, 4(1), 1-16. Recuperado em 13 julho 2009, de http:// www.revistasusp.sibi.usp.br/scielo.php?pid=S180669762008000100005\&script $=$ sci_arttext

Martins, R. A., Manzatto, A. J., Cruz, L. N., Poiate, S. M. G., \& Scarin, A. C. F. (2008). Utilização do alcohol use disordens identification test (AUDIT) para identificação do consumo de álcool entre estudantes do ensino médio [Versão eletrônica]. Revista Interamericana de Psicologia, 42(2), 307-16. Recuperado em 27 outubro 2010, http:// redalyc.uaemex.mx/redalyc/pdf/284/28442212.pdf

Mendez, E. B. (1999). Uma versão brasileira do AUDIT Alcohol Use Disorders Identification Test. Dissertação de mestrado não publicada, Universidade Federal de Pelotas, Pelotas, RS.

Ministério da Saúde. (2004). A política do Ministério da Saúde para atenção integral a usuários de álcool e outras drogas (2a ed.). Brasília: Ministério da Saúde.
Muza, G. M., Bettiol, H., Muccilo, G., \& Barbieri, M. A. (1997). Consumo de substâncias psicoativas por adolescentes escolares de Ribeirão Preto, SP (Brasil): Prevalência do consumo por sexo, idade e tipo de substância. Revista de Saúde Pública, 31(1), 21-29.

Pereira, J. C. R. (1999). Análise de dados qualitativos: Estratégias metodológicas para as ciências de saúde, humanas e sociais. São Paulo: Ed. USP.

Sanches, Z. V. M., \& Nappo, S. A. (2007). A religiosidade, a espiritualidade e o consumo de drogas [Versão eletrônica]. Revista de Psiquiatria Clínica, 34(Supl. 1), 73-81. Recuperado em 16 abril 2009, de http://www. honet.usp.br/ipq/revista/vol34/s1/73.html

Sapienza, G., \& Pedremonico, M. R. (2005). Risco, proteção e resiliência no desenvolvimento da criança e do adolescente [Versão eletrônica]. Psicologia em Estudo, 10(2), 209-216. Recuperado em 19 setembro 2009, de http://www.scielo.br/pdf/pe/v10n2/v10n2a07.pdf

Scivoletto, S., \& Giusti, L. S. (2007). Fatores protetores e de risco associados ao uso de drogas na adolescência [Versão eletrônica]. Álcool e drogas sem distorção. Recuperado em 04 julho de 2009, de http://apps.einstein. br/alcooledrogas/novosite/atualizacoes/ac_131.htm

Silva, V. A., Aguiar, A. S., Felix, F., Rebello, G. P., Andrade, R. C., \& Mattos, H. F. (2003). Estudo brasileiro sobre abuso de substâncias por adolescentes: Fatores associados e adesão ao tratamento [Versão eletrônica]. Revista Brasileira de Psiquiatria, 25(3), 133-138. Recuperado em 07 outubro 2009, de http://www. scielo.br/scielo.php?script=sci_arttext\&pid $=$ S151644462003000300004

Statistical Package for the social Science for Windows - SPSS. (2003). SPSS/Pc version 13.0 [Programa de computador]. Chicago: Author.

Luana Dallo é doutoranda em Educação pelo Programa de Pós-graduação da Faculdade de Filosofia e Ciências da Universidade Estadual Paulista, campus Marília.

Raul Aragão Martins é Professor Adjunto de Psicologia da Educação do Departamento de Educação do Instituto de Biociências, Letras e Ciências Exatas da Universidade Estadual Paulista, campus São José do Rio Preto.

Recebido: $29 / 01 / 2010$

$1^{a}$ revisão: 21/12/2010

Aceite final: 24/01/2011 